\title{
Melatonin and Other 5-Methoxylated Indoles in Yeast: Presence in High Concentrations and Dependence on Tryptophan Availability
}

\author{
J. Sprenger, ${ }^{1}$ R. Hardeland, ${ }^{1}$ B. Fuhrberg ${ }^{1}$ and S. - Z. $\mathrm{Han}^{2}$ \\ ${ }^{1}$ Institut für Zoologie und Anthropologie, Universität Göttingen, Berliner Str. 28, D-37073 Göttingen, Germany \\ ${ }^{2}$ Department of Biology, Hallym University, 1 Okchon-dong, Chunchon 200-702, S-Korea
}

Accepted March 3, 1999

\begin{abstract}
Summary Melatonin (MLT) and two structurally related compounds, 5-methoxytryptamine (5MT) and 5-methoxytryptophol (5 ML), are formed in Saccharomyces cerevisiae in high concentrations. Depending on conditions, levels can attain several $\mathrm{ng} / \mathrm{mg}$ protein, sometimes more than 10 (MLT, $5 \mathrm{MT}$ ) or 20 (up to $>100: 5 \mathrm{ML}$ ) ng/mg. Starvation in salt medium leads to a drop in all methoxyindoles below detection thresholds. After $4 \mathrm{~h}$ in salt, reincubation in growth medium restores high levels. Addition of tryptophan, serotonin or $N$-acetylserotonin to starved cells also elevates MLT and 5MT. Exogenous MLT (50 or $100 \mu \mathrm{M}$ ) causes $5 \mathrm{MT}$ to rise above $700 \mathrm{ng} / \mathrm{mg}$ protein within $0.5 \mathrm{~h}$, whereas same concentrations of exogenous 5MT lead to $>90->300 \mathrm{ng}$ MLT $/ \mathrm{mg}$. Therefore, MLT can be synthesized via the most common pathway from tryptophan to $N$-acetylserotonin as a direct precursor, and is deacetylated to 5MT; biosynthesis of MLT by $N$-acetylation of 5MT is a secondary pathway.
\end{abstract}

Melatonin, originally discovered as the hormone of the pineal gland, has now been detected in various non-vertebrate taxa, including bacteria (Manchester et al. 1995, Tilden et al. 1997), protozoa, uni- and multicellular algae, higher plants and various invertebrate animals (review: Hardeland and Fuhrberg 1996). While melatonin's role has been identified in vertebrates as that of a chemical messenger conveying the signal darkness (Reiter 1991, 1993), with implications in photoperiodic time measurement and phase control of circadian rhythms, much less is known on its role in other organisms. Only in the dinoflagellate Gonyaulax polyedra, the mimicking of photoperiodic effects (Balzer and Hardeland 1991, Hardeland 1993) and strong responses to temperature changes (Fuhrberg et al. 1997) have been described. It was, however, assumed that the primary role of melatonin might be different from that of a mediator of darkness, perhaps relating to its properties as a scavenger of free-radicals (Hardeland et al. 1995). This would be in accordance with the high levels of melatonin found in various unicellular organisms (Hardeland and Fuhrberg 1996) and the demonstrable protection from oxidative stress by physiologically possible concentrations in the dinoflagellate mentioned (Antolín et al. 1997). To date, no detailed informations on melatonin in fungi have been available (Hardeland et al. 1996). In this study on Saccharomyces cerevisiae, we have investigated an organism in which circadian behaviour has not been demonstrated and may be absent. Presence of melatonin would indicate a physiological function different from mediation of dark signals and readjustment of circadian oscillators.

Commercially available baker's yeast was suspended at cell densities of 10 or $1 \mathrm{mg} / \mathrm{ml}$ (cf. Tables and legend of Fig. 1), in either standard growth medium ( $20 \mathrm{~g}$ peptone $/ 1,20 \mathrm{~g}$ glucose $/ 1,10 \mathrm{ml}$ yeast extract/l) or salt medium ( $50 \mathrm{mM} \mathrm{Na}_{2} \mathrm{HPO}_{4}, 0.5 \% \mathrm{NaCl}$, adjusted to $\mathrm{pH} 5.8$, using citric acid). Cells were incubated in Erlenmeyer flasks at $25^{\circ} \mathrm{C}$, in complete darkness for the entire period of incubation, and kept in suspension by continuous shaking. For duration of incubations see Figure legend and Tables. In the experiments reported here, flasks were closed by air-permeable stoppers. In 
Table 1. Effects of growth conditions on levels of 5-methoxyindoles

\begin{tabular}{|c|c|c|c|c|c|}
\hline \multirow{2}{*}{ Medium } & \multirow{2}{*}{$\begin{array}{c}\text { Incubation } \\
{[\mathrm{h}]}\end{array}$} & \multirow{2}{*}{$\begin{array}{l}\text { Cell density } \\
{[\mathrm{mg} / \mathrm{ml}]}\end{array}$} & \multicolumn{3}{|c|}{ Concentrations of metabolites $[\mathrm{ng} / \mathrm{mg}$ protein $] \pm$ s.e.m. } \\
\hline & & & MLT & $5 \mathrm{MT}$ & $5 \mathrm{ML}$ \\
\hline Growth m. & 2 & 10 & $94.7 \pm 66.5$ & $34.8 \pm 27.5$ & $5.2 \pm 1.4$ \\
\hline Growth $\mathrm{m}$. & 4 & 10 & $6.1 \pm 0.6$ & $6.9 \pm 5.2$ & $5.3 \pm 0.5$ \\
\hline Growth m. & 6 & 10 & $5.6 \pm 1.4$ & $4.1 \pm 2.5$ & $5.3 \pm 1.7$ \\
\hline Growth m. & 2 & 1 & $16.9 \pm 5.6$ & $5.8 \pm 2.8$ & $26.4 \pm 17.0$ \\
\hline Growth m. & 4 & 1 & $9.2 \pm 3.2$ & $3.2 \pm 1.5$ & $18.5 \pm 10.4$ \\
\hline Growth m. & 6 & 1 & $9.3 \pm 2.9$ & $3.3 \pm 1.9$ & $21.9 \pm 10.4$ \\
\hline Salt m. & 4 & 1 & n.dtcd. & n.dtcd. & n.dtcd. \\
\hline Salt m. & 6 & 1 & n.dtcd. & n.dtcd. & n.dtcd. \\
\hline Salt $\mathrm{m}$. & 4 & & & & \\
\hline + growth $\mathrm{m}$ & 0.5 & 1 & $25.7 \pm 9.0$ & n.dtcd. & $24.3 \pm 9.1$ \\
\hline Salt $\mathrm{m}$. & 4 & & & & \\
\hline + growth $\mathrm{m}$. & 1 & 1 & $18.9 \pm 7.4$ & n.dtcd. & $26.0 \pm 10.6$ \\
\hline Salt $\mathrm{m}$ & 4 & & & & \\
\hline + growth $\mathrm{m}$ & 6 & 1 & $6.4 \pm 2.3$ & $9.3 \pm 9.3$ & $13.3 \pm 6.1$ \\
\hline Salt $\mathrm{m}$. & 4 & & & & \\
\hline + growth $\mathrm{m}$ & 8 & 1 & $3.3 \pm 1.1$ & $1.0 \pm 1.0$ & $10.6 \pm 3.7$ \\
\hline Salt m. & 4 & & & & \\
\hline + growth m. & 10 & 1 & $2.4 \pm 1.6$ & $0.5 \pm 0.5$ & $11.7 \pm 3.1$ \\
\hline Salt $\mathrm{m}$ & 4 & & & & \\
\hline + growth $\mathrm{m}$ & 12 & 1 & $1.7 \pm 1.1$ & $0.3 \pm 0.3$ & $12.5 \pm 2.2$ \\
\hline
\end{tabular}

m. = medium; .dtcd. $=$ not detected.

Table 2. Effects of tryptophan and indoleamines in cells depleted of 5-methoxyindoles by a passage through salt medium. Cells $(1 \mathrm{mg} / \mathrm{ml})$ were incubated for $4 \mathrm{~h}$ in salt medium, in order to decrease 5-methoxyindole concentrations to undetectable levels; thereafter, tryptophan or one of its metabolites was added and methoxyindoles were determined 15 or 30 min later. $5 \mathrm{ML}$ was usually undetectable after this short incubation period

\begin{tabular}{|c|c|c|c|}
\hline \multirow{2}{*}{$\begin{array}{l}\text { Substance added } \\
\text { after } 4 \mathrm{~h} \text { in salt } \mathrm{m} \text {. }\end{array}$} & \multirow{2}{*}{$\begin{array}{c}\text { Incubation } \\
\text { with indolic } \\
\text { metabolite } \\
\text { [min] }\end{array}$} & \multicolumn{2}{|c|}{$\begin{array}{l}\text { Concentrations of 5-methoxylated indoleamines } \\
{[\mathrm{ng} / \mathrm{mg} \text { protein }] \pm \text { s.e.m. }}\end{array}$} \\
\hline & & MLT & $5 \mathrm{MT}$ \\
\hline Tryptophan $1 \mathrm{mM}$ & 30 & $25.3 \pm 10.9$ & $305.3 \pm 18.6$ \\
\hline Serotonin $1 \mathrm{mM}$ & 15 & $18.0 \pm 12.0$ & n.dtcd. \\
\hline Serotonin $1 \mathrm{mM}$ & 30 & $8.5 \pm 4.6$ & $234.1 \pm 82.1$ \\
\hline$N$-Acetylserotonin $1 \mathrm{mM}$ & 15 & $43.1 \pm 32.8$ & n.dtcd. \\
\hline$N$-Acetylserotonin $1 \mathrm{mM}$ & 30 & n.dtcd. & $201.7 \pm 14.7$ \\
\hline Melatonin 0.05 or $0.1 \mathrm{mM}$ & 30 & exog. & $>700->1000$ \\
\hline $5 \mathrm{MT} 0.05$ or $0.1 \mathrm{mM}$ & 30 & $>90->300$ & exog. \\
\hline
\end{tabular}

n.dtcd. $=$ not detected; exog. $=$ exogenous (therefore, determination would only reflect the concentration of the substance taken up from the medium).

other experiments (not shown), we had also investigated the possible influences of fermentation under occlusion from oxygen and of presence of 5\% ethanol. None of these other treatments led to substantial deviations in methoxyindole concentrations. In further experiments (Table 1), we subjected cells to a passage through salt, followed by incubation in growth medium. In these cases, an equal volume of double-concentrated growth medium was added. Indolic compounds were also added to cell suspensions kept in salt medium (for concentrations see Table 2). Tryptophan was directly dissolved in salt medium; indoleamines were pre-dissolved in concentrations of $0.2 \mathrm{M}$ in DMSO; because of light sensitivity of these compounds, the entire procedure was carried out under 


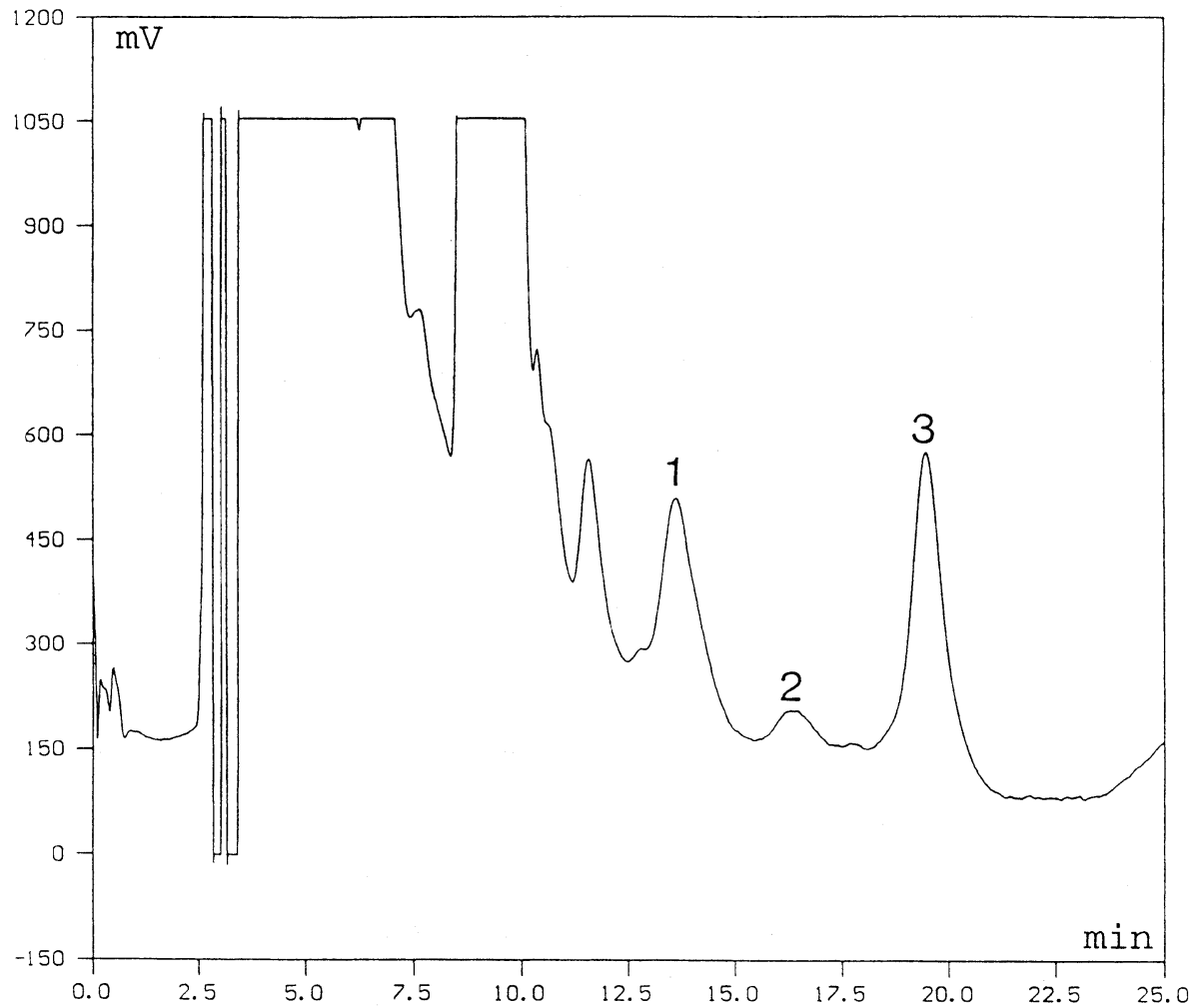

Fig. 1. HPLC chromatogram of yeast extract showing the presence of three 5-methoxyindoles by electrochemical detection. 1) 5ML, 2) 5MT, 3) MLT. Cells $(1 \mathrm{mg} / \mathrm{ml})$ had been incubated for $4 \mathrm{~h}$ in growth medium. Before injection, the deproteinized extract had been diluted 1:10 with eluent. Ordinate: electrochemical signal; abscissa: retention time.

dim red light (Balzer and Hardeland 1991).

Extraction of cells was also performed under red light. After incubation, cells were collected by centrifuging $10 \mathrm{ml}$ samples ( $10 \mathrm{~min}$ at $4,000 \mathrm{rpm}$, Hettich EBA $3 \mathrm{~S}$ ). Pellets were resuspended in $3 \mathrm{ml}$ of $0.6 \mathrm{M} \mathrm{Tris} / \mathrm{HCl}$ buffer, $\mathrm{pH} 8.4$, shock-frozen in liquid nitrogen, pulverized in a mortar, stored at $-80^{\circ} \mathrm{C}$, and extracted with an equal volume of $0.4 \mathrm{~N}$ perchloric acid; after $15 \mathrm{~min}$ at $4{ }^{\circ} \mathrm{C}$, protein precipitates were removed by 2 centrifugations for $10 \mathrm{~min}$ at $12,000 \times \mathrm{g}$. This particularly preservative procedure (Poeggeler et al. 1991, Hardeland and Poeggeler 1994) turned out to be very efficient in Saccharomyces: recovery rates, as determined after addition of exogenous methoxyindoles, were usually between 84 and $90 \%$.

Concentrations of methoxyindoles were measured by reversed-phase HPLC with electrochemical detection, according to Poeggeler et al. (1991), using the modification and equipment as described by Fuhrberg et al. (1996). The oxidation potential applied was $800 \mathrm{mV}$. Protein was determined according to Lowry et al. (1951).

\section{Results and discussion}

Saccharomyces cerevisiae is the first fungus in which the presence of melatonin (MLT) is demonstrated (Fig. 1, Table 1). Moreover, two other methoxyindoles are detected which are frequently associated with melatonin in unicellular organisms (Hardeland and Fuhrberg 1996, Fuhrberg et al. 1997), namely, 5-methoxytryptamine (5MT) and 5-methoxytryptophol (5ML). In an earlier study (Kubis et al. 1992), MLT had been shown to alter the circadian rhythm of $\mathrm{NAD}^{+} \mathrm{ki}^{-}$ 
nase in Neurospora crassa. Since, however, circadian rhythms are not demonstrable in Saccharomyces cerevisiae and presumably absent in this organism, melatonin's role in yeast should be different from that of controlling rhythmicity and, most likely, from that of a dark signal. To date, we have not found any sign of circadian melatonin rhythm in yeast. Moreover, concentrations of melatonin and the other methoxyindoles can be fairly high (Table 1), exceeding by orders of magnitude the levels found in vertebrate blood. With regard to the large amounts melatonin attains in yeast, other functions such as participation in antioxidative protection seem possible, as suggested earlier for dinoflagellates (Hardeland 1993, Hardeland et al. 1995, 1996, Antolín et al. 1997).

In $S$. cerevisiae, levels of methoxyindoles can be influenced by growth conditions (Table 1). Changes depending on cell density and duration of incubation are still difficult to interpret. With regard to a considerable variation between cultures prepared from different material, these alterations should not be overrated, at the present state of our understanding. However, a very dramatic change in methoxyindole concentrations was observed when cells were incubated in salt medium: Within $4 \mathrm{~h}$, the three indolic compounds dropped to undectable levels (Table 1). After a $4 \mathrm{~h}$ passge through salt, incubation in growth medium resulted in a rapid reappearance of melatonin and 5ML, whereas $5 \mathrm{MT}$ was detected later. With extended incubation time, methoxyindole concentrations gradually declined again.

In further experiments, the causes for the disappearance of methoxyindoles under starvation were investigated. The drop of 5-methoxyindoles in salt medium does not seem to reflect down-regulation of enzymes of melatonin formation by general mechanisms related to growth control, but rather deficiency of the precursor, tryptophan. After addition of this amino acid to starved cells, melatonin, and 5MT as well, rose within $30 \mathrm{~min}$ to remarkably high levels (Table 2). In particular, $5 \mathrm{MT}$ was elevated to concentrations otherwise not found under normal growth conditions.

Within the relatively short period of exposure to tryptophan $(30 \mathrm{~min}), 5 \mathrm{ML}$ did not increase to safely detectable levels in these experiments, nor in those with other potential melatonin precursors (no data presented). Thus, the major pathway of 5ML formation of 5ML remains to be identified. On the one hand, this substance may be generated via 5MT oxidation by monoamine oxidase and subsequent reduction of the resulting indolic aldehyde by alcohol dehydrogenase; alternatively, the indolic alcohol may also be formed from 5-methoxytryptophan or by $O$-methylation of the serotonin metabolite, 5-hydroxytryptophol (Hardeland et al. 1993). With regard to the low levels of $5 \mathrm{ML}$ in the precursor supplementation experiments, and to the presence of relatively high $5 \mathrm{ML}$ concentrations in some other experiments in which 5MT remained undetectable (Table 1), it should be concluded that the formation from 5MT is not the prevailing pathway.

Results similar to those after tryptophan supplementation were obtained upon addition of serotonin, another precursor in the most common biosynthetic pathway of melatonin. In this case, a characteristic dependence on incubation time was observed (Table 2): After $15 \mathrm{~min}$ in the presence of serotonin, melatonin was already detectable, but not 5MT, whereas after $30 \mathrm{~min}$, 5MT had increased to very high concentrations. Incubation with $\mathrm{N}$-acetylserotonin, representing in other organisms the direct precursor of melatonin, led to similar results: Melatonin was found in high concentrations already after $15 \mathrm{~min}$, whereas $5 \mathrm{MT}$ appeared after $30 \mathrm{~min}$, while melatonin declined again below the determination threshold. Correspondingly, the addition of melatonin led, within $30 \mathrm{~min}$, to very high levels of 5MT. On the other hand, incubation with 5MT resulted in large amounts of melatonin.

Taken together, these results demonstrate that melatonin can be formed in Saccharomyces from precursors of the normal biosynthetic pathway, as known from other organisms, i.e., from tryptophan via 5-hydroxytryptophan, serotonin and $N$-acetylserotonin. 5MT, which appeared after melatonin, in experiments of supplementation with both full growth medium (Table 1) and precursors (Table 2), and which was detected in very high concentrations after addition of melatonin, can obviously be formed by deacetylation of melatonin. However, since melatonin can also be generat- 
ed from 5MT, an alternative biosynthetic pathway is likewise possible, in terms of a side shunt, namely, $O$-methylation of serotonin followed by $N$-acetylation of 5MT. This conclusion reminds strongly of the situation in another lower eucaryote, the dinoflagellate Gonyaulax polyedra, in which melatonin is predominantly formed from $N$-acetylserotonin, but also from 5MT if serotonin is available at high concentrations or when the activity of the melatonin-deacetylating aryl acylamidase is low (Hardeland 1993, Hardeland and Fuhrberg 1996).

\section{References}

Antolín, I., Obst, B., Burkhardt, S. and Hardeland, R. 1997. Antioxidative protection in a high-melatonin organism: The dinoflagellate Gonyaulax polyedra is rescued from lethal oxidative stress by strongly elevated, but physiologically possible concentrations of melatonin. J. Pineal Res. 23: 182-190.

Balzer, I. and Hardeland, R. 1991. Photoperiodism and effects of indoleamines in a unicellular alga, Gonyaulax polyedra. Science 253: 795-797.

Fuhrberg, B., Balzer, I., Hardeland, R., Werner, A. and Lüning, K. 1996. The vertebrate pineal hormone melatonin is produced by the brown alga Pterygophora californica and mimics dark effects on growth rate in the light. Planta 200: 125-131.

-, Hardeland, R., Poeggeler, B. and Behrmann, G. 1997. Dramatic rises of melatonin and 5-methoxytryptamine in Gonyaulax exposed to decreased temperature. Biol. Rhythm Res. 28: 144-150.

Hardeland, R. 1993. The presence and function of melatonin and structurally related indoleamines in a dinoflagellate, and a hypothesis on the evolutionary significance of these tryptophan metabolites in unicellulars. Experientia 49: $614-622$.

—, Balzer, I., Fuhrberg, B. and Antolín, I. 1996. Melatonin and Other Methoxyindoles in Non-vertebrates. In: Hardeland, R. (ed.) Metabolism and Cellular Dynamics of Indoles. Univ. of Göttingen, Göttingen, pp. 162-172.

—, - , Poeggeler, B., Fuhrberg, B., Uría, H., Behrmann, G., Wolf, R., Meyer, T. J. and Reiter, R. J. 1995. On the primary functions of melatonin in evolution: Mediation of photoperiodic signals in a unicell, photooxidation and scavenging of free radicals. J. Pineal Res. 18: 104-111.

- and Fuhrberg, B. 1996. Ubiquitous melatonin-Presence and effects in unicells, plants and animals. Trends Comp. Biochem. Physiol. 2: 25-45.

-, Reiter, R. J., Poeggeler, B. and Tan, D.-X. 1993. The significance of the metabolism of the neurohormone melatonin: antioxidative protection and formation of bioactive substances. Neurosci. Biobehav. Rev. 17: 347-357.

Kubis, H.-P., Balzer, I. and Hardeland, R. 1992. Effects of 1,2-dihydro-4-hydroxy-6-methoxy-N-methyl quinoline in relation to circadian rhythms of $\mathrm{NAD}^{+}$kinase and $\mathrm{NADP}^{+}$autoreduction in Neurospora crassa and bioluminescence in Gonyaulax polyedra. Comp. Biochem. Physiol. 102C: 97-101.

Lowry, O. H., Rosebrough, N. J., Farr, A. L. and Randall, R. J. 1951. Protein measurement with the Folin phenol reagent. J. Biol. Chem. 193: 265-275.

Manchester, L. C., Poeggeler, B., Alvares, F. L., Ogden, G. B. and Reiter, R. J. 1995. Melatonin immunoreactivity in the photosynthetic prokaryote, Rhodospirillum rubrum: Implications for an ancient antioxidant system. Cell. Molec. Biol. Res. 41: 391-395.

Poeggeler, B., Balzer, I., Hardeland, R. and Lerchl, A. 1991. Pineal hormone melatonin oscillates also in the dinoflagellate Gonyaulax polyedra. Naturwissenschaften 78: 268-269.

— and Hardeland, R. 1994. Detection and quantification of melatonin in a dinoflagellate, Gonyaulax polyedra. Solutions to the problem of methoxyindole destruction in non-vertebrate material. J. Pineal Res. 17: 1-10.

Reiter, R. J. 1991. Melatonin: The chemical expression of darkness. Molec. Cell Endocrinol. 79: C153-C159.

- 1993. The melatonin rhythm: both a clock and a calendar. Experientia 49: 654-664.

Tilden, A. R., Becker, M. A., Amma, L. L., Arciniega, J. and McGraw, A. K. 1997. Melatonin production in an aerobic photosynthetic bacterium: An evolutionarily early association with darkness. J. Pineal Res. 22: 102-106. 